\title{
Correction to: Metabolic Brain Network Analysis of Hypothyroidism Symptom Based on $\left[{ }^{18} \mathrm{~F}\right] \mathrm{FDG}$-PET of Rats
}

Hongkai Wang, ${ }^{2}$ Ziyu Tan, ${ }^{2}$ Qiang Zheng, ${ }^{1}$ Jing $\mathrm{Yu}^{1}$

${ }^{1}$ Nuclear Medicine Division, Second Affiliated Hospital, Dalian Medical University, Dalian, 116023, Liaoning, China

${ }^{2}$ Department of Biomedical Engineering, Dalian University of Technology, Dalian, 116024, Liaoning, China

Correction to: Mol Imaging Biol (2018).

https://doi.org/10.1007/s11307-018-1182-2

This article was updated to correct the spelling of Hongkai Wang's name: it is correct as displayed here. The original article has been corrected.

The online version of the original article can be found at https://doi.org/ $10.1007 / \mathrm{s} 11307-018-1182-2$

Correspondence to: Qiang Zheng; e-mail: Oliver951101@outlook.com 